Research Article

\title{
Exact Analytical Solutions of Generalized Fifth-Order KdV Equation by the Extended Complex Method
}

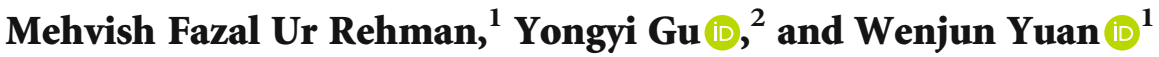 \\ ${ }^{1}$ School of Mathematics and Information Science, Guangzhou University, Guangzhou 510006, China \\ ${ }^{2}$ Big Data and Educational Statistics Application Laboratory, Guangdong University of Finance and Economics, \\ Guangzhou 510320, China
}

Correspondence should be addressed to Yongyi Gu; gdguyongyi@163.com and Wenjun Yuan; wjyuan1957@126.com

Received 31 January 2021; Revised 23 March 2021; Accepted 5 April 2021; Published 8 May 2021

Academic Editor: Chuanjun Chen

Copyright (c) 2021 Mehvish Fazal Ur Rehman et al. This is an open access article distributed under the Creative Commons Attribution License, which permits unrestricted use, distribution, and reproduction in any medium, provided the original work is properly cited.

\begin{abstract}
The recently introduced technique, namely, the extended complex method, is used to explore exact solutions for the generalized fifth-order KdV equation. Appropriately, the rational, periodic, and elliptic function solutions are obtained by this technique. The 3D graphs explain the different physical phenomena to the exact solutions of this equation. This idea specifies that the extended complex method can acquire exact solutions of several differential equations in engineering. These results reveal that the extended complex method can be directly and easily used to solve further higher-order nonlinear partial differential equations (NLPDEs). All computer simulations are constructed by maple packages.
\end{abstract}

\section{Introduction}

In the 20 century, nonlinear science (NLS) plays a significant role in special inventions, for example, the invention of the radio, the discovery of DNA structure for biology, the development of quantum theory for theoretical physics and chemistry, and the invention of transister for computer engineering. It is well known that NLS belongs to the NLPDEs which are introduced in several areas such as fluid thermodynamics, plasma diffusion, biology, physics, geometry, and population dynamics.

Lots of studies are focused on the differential equations [1-10], and many effective techniques are used to acquire analytical and numerical solutions for NLPDEs such as sine-cosine method [11], extended sinh-Gorden equation expansion method [12], variation iteration algorithm [13], homotopy perturbation method [14], F-expansion method [15], Exp-function expansion method [16], first integral method [17], Ansatz method [18], generalized Kudryashov method [19], $\left(G^{\prime} / G\right)$-expansion method [20], projective Riccati equation method [21], tanh method [22], nonpolynomial spline method [23], B-spline method [24], B-spline colloca- tion [25], Weierstrass elliptic function method [26], Laplace decomposition method [27], extended direct algebraic method [28, 29], Sub-ODE method [30], Darboux transformation [31], and extended tanh-coth method [32, 33]. The generalized fifth-order KdV equation [34] is represented by

$$
w_{t}+s w w_{x}+f w^{2} w_{x}+e w_{x x x}+\mu w_{x x x x x}=0,
$$

where $s, f, e$, and $\mu$ are the arbitrary constants. This equation is a nonlinear model in many long wave physical phenomena. It is used in the shallow water wave with surface tension and magnetoacoustic wave in plasma. Several researchers have explored the analytical solutions of generalized fifth-order KdV equation such as Hedli and Kadem have attained a new analytical solution for the fifth-order $\mathrm{KdV}$ equation by the exponential expansion method [35]. Dinarvand et al. have found approximate analytical solutions of the sawada-kotera and Lax's fifth-order KdV equations by homotopy analysis technique [36]. Salas and Lugo have introduced extended tanh method to obtain the exact solutions of the general fifth-order KdV equation [37]. Alam and Xin et al. have attained new exact 
solutions by $\left(G^{\prime} / G\right)$-expansion method of modified KdVZakharov-Kuznetsov equation [38]. Ganji and Abdollahzadeh have introduced the sech method and rational expfunction method to find the exact traveling wave solutions of the Lax's seventh-order KdV equation [39].

In the present work, our main purpose is to calculate the generalized fifth-order $\mathrm{KdV}$ equation by the extended complex method based on the concept of Yuan et al. [40-46]. It is a remarkable approach to attain exact analytical solutions. Our technique would be potentially applied to various processes of the engineering field. This article is organized as mentioned as follows. In Section 2, methods and materials are described. In Section 3, the application of the introduced method is determined. Section 4 deals with physical phenomena of important results. The comparison and conclusions are explained in Section 5.

\section{Methods and Materials}

Let us consider the general form of NLPDE

$$
l\left(w, w_{t}, w_{x}, w_{z}, w_{t t}, w_{x x} \ldots \ldots \ldots\right),
$$

where the unknown function is $w=w(x, t)$ and $l$ is a polynomial in $w=w(x, t)$ and its derivatives.

Step 1. A transformation $T: w(x, t) \rightarrow W(z)$ is introduced, and $(x, t)$ can be introduced in different standard; hence, we have used the transformation such as

$$
w(x, t)=W(z), z=k(x-\omega t) .
$$

Step 2. The $w(x, t)=W(z), z=k(x-\omega t)$ transform Eq. (2) into nonlinear ODE:

$$
T\left(W, W^{\prime}, W^{\prime \prime}, W^{\prime \prime \prime}, \cdots\right)=0,
$$

in Eq. (4), where $W$ primes are the derivatives w.r.t $z$. This equation is reduced by further integration.

Step 3. Let the meromorphic solutions $W$ of Eq. (4) have at least one pole, and let us consider $p, q \in \mathbb{Z}$. For this condition, we substitute the Laurent series

$$
W(z)=\sum_{k=-q}^{\infty} B_{k} z^{k}, q>0, B_{-q} \neq 0
$$

into Eq. (4), if we can find $p$ distinct Laurent singular parts:

$$
\sum_{k=-q}^{-1} B_{k} z^{k}
$$

then the weak $\langle p, q\rangle$ condition of Eq. (4) holds. Weierstrass elliptic function $\wp(z):=\wp\left(z, g_{2}, g_{3}\right)$ with double periods of the equation is given as below:

$$
\left(\wp^{\prime}(z)\right)^{2}=4 \wp(z)^{3}-g_{2} \wp(z)-g_{3}
$$

and the addition formula is mentioned as below:

$$
\wp\left(z-z_{0}\right)=-\wp(z)+\frac{1}{4}\left[\frac{\wp^{\prime}(z)+\wp^{\prime}\left(z_{0}\right)}{\wp(z)-\wp\left(z_{0}\right)}\right]^{2}-\wp\left(z_{0}\right) .
$$

Step 4. Putting the indeterminate forms

$$
\begin{aligned}
W(z)= & \sum_{i=1}^{y-1} \sum_{j=2}^{q} \frac{(-1)^{j} \delta_{-i j}}{(j-1) !} \frac{d^{j-2}}{d z^{j-2}}\left(\frac{1}{4}\left[\frac{\wp^{\prime}(z)+G_{i}}{\mathfrak{\wp}(z)-H_{i}}\right]^{2}-\wp(z)\right) \\
& +\sum_{i=1}^{y-1} \frac{\delta_{-i 1}}{2} \frac{\wp^{\prime}(z)+G_{i}}{\wp(z)-H_{i}}+\sum_{j=2}^{q} \frac{(-1)^{j} \delta_{-y j}}{(j-1) !} \frac{d^{j-2}}{d z^{j-2}} \wp(z)+\delta_{0},
\end{aligned}
$$

$$
\begin{gathered}
W(z)=\sum_{i=1}^{y} \sum_{j=1}^{q} \frac{\delta_{i j}}{\left(z-z_{i}\right)^{j}}+\delta_{0}, \\
W\left(e^{\alpha z}\right)=\sum_{i=1}^{y} \sum_{j=1}^{q} \frac{\delta_{i j}}{\left(e^{\alpha z}-e^{\alpha z_{i}}\right)^{j}}+\delta_{0},
\end{gathered}
$$

into Eq. (4); hence, the number of equations is computed by adjusting the coefficient to zero. These algebraic equations are calculated by the source of maple. Equation (9) is the elliptic solution $W$ with pole at $z=0$, where $\delta_{-i j}$ are attained by (4), $G_{i}^{2}=4 H_{i}^{3}-g_{2} H_{i}-g_{3}, \sum_{i=1}^{y} \delta_{-i 1}=0$. Equation (10) is the rational function, and Eq. (11) is the exponential function which are denoted as $W(z), W\left(e^{\alpha z}\right)(\alpha \in \mathbb{C})$, and they have $y$ $(\leq p)$ distinct poles of multiplicity $q$.

Step 5. The meromorphic solutions are got with the arbitrary pole. Substitute inverse transformation $T^{-1}$ into meromorphic solutions; then, we obtain the exact analytical solutions of NLPDEs.

\section{Application of the Method}

In this section, we would like to find the exact analytical solutions of a generalized fifth-order KdV equation by extended complex approach. Substitute

$$
w(x, t)=W(z), z=k(x-\omega t)
$$

into Eq. (1), then obtain

$$
-\omega W^{\prime}+s W W^{\prime}+f W^{2} W^{\prime}+e k^{2} W^{\prime \prime \prime}+\mu k^{4} W^{\prime \prime \prime \prime \prime}=0,
$$


now, we integrate Eq. (13) w.r.t $z$; then, we attain new ODE

$$
-\omega W+s \frac{W^{2}}{2}+f \frac{W^{3}}{3}+e k^{2} W^{\prime \prime}+\mu k^{4} W^{\prime \prime \prime \prime}=0
$$

Putting (5) into (14) then we have $p=1$ and $q=2$; hence, the weak $\langle 1,2\rangle$ condition of (14) holds. By weak \langle $1,2\rangle$ and (10), then rational solutions with pole at $z=0$ are

$$
W_{r}(z)=\frac{\delta_{12}}{(z-1)^{2}}+\frac{\delta_{11}}{z-1}+\delta_{10}
$$

substituting the $W_{r}(z)$ into Eq. (14); then, we have

$$
\frac{1}{6} \sum_{i=1}^{7} c_{1 i} z^{(7-i)}(z-1)^{-6}=0
$$

where

$$
\begin{aligned}
& c_{11}=2 \delta_{10}^{3} f+3 \delta_{10}^{2} s-6 \delta_{10} \omega, \\
& c_{12}=6 \delta_{11} \delta_{10}^{2} f-12 \delta_{10}^{3} f+6 \delta_{11} \delta_{10} s-18 \delta_{10}^{2} s-6 \delta_{11} \omega+36 \delta_{10} \omega, \\
& c_{13}=6 \delta_{12} \delta_{10}^{2} f+6 \delta_{11}^{2} \delta_{10} f-30 \delta_{11} \delta_{10}^{2} f+30 \delta_{10}^{3} f+6 \delta_{12} \delta_{10} s \\
& +3 \delta_{11}^{2} s-30 \delta_{11} \delta_{10} s+45 \delta_{10}^{2} s-6 \delta_{12} \omega+30 \delta_{11} \omega-90 \delta_{10} \omega, \\
& c_{14}=12 \delta_{12} \delta_{11} \delta_{10} f-24 \delta_{12} \delta_{10}^{2} f+2 \delta_{11}^{3} f-24 \delta_{11}^{2} \delta_{10} f+60 \delta_{11} \delta_{10}^{2} f \\
& +12 \delta_{11} e k^{2}-40 \delta_{10}^{3} f+6 \delta_{12} \delta_{11} s-24 \delta_{12} \delta_{10} s-12 \delta_{11}^{2} s \\
& +60 \delta_{11} \delta_{10} s-60 \delta_{12}^{2} s+24 \delta_{12} \omega-60 \delta_{11} \omega+120 \delta_{10} \omega \text {, } \\
& c_{15}=6 \delta_{12}^{2} \delta_{10} f+6 \delta_{12} \delta_{11}^{2} f-36 \delta_{12} \delta_{11} \delta_{10} f+36 \delta_{12} \delta_{10}^{2} f+36 \delta_{12} e k^{2} \\
& -6 \delta_{11}^{3} f+36 \delta_{11}^{2} \delta_{10} f-60 \delta_{11} \delta_{10}^{2} f-36 \delta_{11} e k^{2}+30 \delta_{10}^{3} f+3 \delta_{12}^{2} s \\
& -18 \delta_{12} \delta_{11} s+36 \delta_{12} \delta_{10} s+18 \delta_{11}^{2} s-60 \delta_{11} \delta_{10} s+45 \delta_{10}^{2} s \\
& -36 \delta_{12} \omega+60 \delta_{11} \omega-90 \delta_{10} \omega \text {, } \\
& c_{16}=144 \delta_{11} k^{4} \mu+6 \delta_{12}^{2} \delta_{11} f-12 \delta_{12}^{2} \delta_{10} f-12 \delta_{12} \delta_{11}^{2} f+36 \delta_{12} \delta_{11} \delta_{10} f \\
& -24 \delta_{12} \delta_{10}^{2} f-72 \delta_{12} e k^{2}+6 \delta_{11}^{3} f-24 \delta_{11}^{2} \delta_{10} f+30 \delta_{11} \delta_{10}^{2} f \\
& +36 \delta_{11} e k^{2}-12 \delta_{10}^{3} f-6 \delta_{12}^{2} s+18 \delta_{12} \delta_{11} s-24 \delta_{12} \delta_{10} s \\
& -12 \delta_{11}^{2} s+30 \delta_{11} \delta_{10} s-18 \delta_{10}^{2} s+24 \delta_{12} \omega-30 \delta_{11} \omega+36 \delta_{10} \omega, \\
& c_{17}=720 \delta_{12} k^{4} \mu-144 \delta_{11} k^{4} \mu+2 \delta_{12}^{3} f-6 \delta_{12}^{2} \delta_{11} f+6 \delta_{12}^{2} \delta_{10} f \\
& +6 \delta_{12} \delta_{11}^{2} f-12 \delta_{12} \delta_{11} \delta_{10} f+6 \delta_{12} \delta_{10}^{2} f+36 \delta_{12} e k^{2} \\
& -2 \delta_{11}^{3} f+6 \delta_{11}^{2} \delta_{10} f-6 \delta_{11} \delta_{10}^{2} f-12 \delta_{11} e k^{2}+2 \delta_{10}^{3} f \\
& +3 \delta_{12}^{2} s-6 \delta_{12} \delta_{11} s+6 \delta_{12} \delta_{10} s+3 \delta_{11}^{2} s-6 \delta_{11} \delta_{10} s \\
& +3 \delta_{10}^{2} s-6 \delta_{12} \omega+6 \delta_{11} \omega-6 \delta_{10} \omega \text {. }
\end{aligned}
$$

By assuming that the coefficients of same powers concerning $z$ in Eq. (16) are zero, then we have numbers of equations:

$$
c_{1 i}=0,(i=1,2, \cdots, 7) .
$$

By solving number of these equations, we obtain

$$
\delta_{12}=-\frac{6 \sqrt{10} \sqrt{\mu} k^{2}}{\sqrt{f}}, \delta_{11}=0, \delta_{10}=0,
$$

then

$$
W_{r 10}(z)=-\frac{6 \sqrt{10} \sqrt{\mu} k^{2} / \sqrt{f}}{(z-1)^{2}}
$$

where $\omega=0$ and $s=-(1 / 5)(\sqrt{f} e \sqrt{10} / \sqrt{\mu})$;

$$
\delta_{12}=\frac{24 k^{2} e}{s}, \delta_{11}=0, \delta_{10}=\frac{6}{5} \frac{e^{2}}{s \mu}
$$

then

$$
W_{r 20}(z)=\frac{24 k^{2} e / s}{(z-1)^{2}}+\frac{6}{5} \frac{e^{2}}{s \mu}
$$

where $f=-(5 / 8)\left(\mu s^{2} / e^{2}\right)$ and $\omega=(3 / 10)\left(e^{2} / \mu\right)$.

$W(z)=R(\eta)$ is a rational function of $\eta=e^{\alpha z}(\alpha \in \mathbb{C})$, applying it into Eq. (14) then

$$
\begin{aligned}
-\omega R & +s \frac{R^{2}}{2}+f \frac{R^{3}}{3}+k^{2} e \alpha^{2}\left(R^{\prime \prime} \eta^{2}+R^{\prime} \eta\right) \\
& +k^{4} \alpha^{4} \mu\left(R^{(4)} \eta^{4}+6 R^{\prime \prime \prime} \eta^{3}+7 R^{\prime \prime} \eta^{2}+R^{\prime} \eta\right)=0,
\end{aligned}
$$

substituting

$$
W_{s}(z)=\frac{\delta_{12}}{(\eta-1)^{2}}+\frac{\delta_{11}}{\eta-1}+\delta_{10}
$$

into the Eq. (23), we attain that

$$
\frac{1}{6} \sum_{i=1}^{7} \frac{c_{2 i} \alpha^{2} \eta^{7-i}}{(\eta-1)^{6}}=0
$$

where

$$
c_{21}=2 \delta_{10}^{3} f+3 \delta_{10}^{2} s-6 \delta_{10} \omega,
$$




$$
\begin{aligned}
c_{22}= & 6 \alpha^{4} \delta_{11} k^{4} \mu+6 \alpha^{2} \delta_{11} e k^{2}+6 \delta_{11} \delta_{10}^{2} f \\
& -12 \delta_{10}^{3} f+6 \delta_{11} \delta_{10} s-18 \delta_{10}^{2} s-6 \delta_{11} \omega+36 \delta_{10} \omega \\
c_{23}= & 96 \delta_{12} \alpha^{4} k^{4} \mu+60 \alpha^{4} \delta_{11} k^{4} \mu+24 \delta_{12} \alpha^{2} e k^{2}-12 \alpha^{2} \delta_{11} e k^{2} \\
& +6 \delta_{12} \delta_{10}^{2} f+6 \delta_{11}^{2} \delta_{10} f-30 \delta_{11} \delta_{10}^{2} f+30 \delta_{10}^{3} f+6 \delta_{12} \delta_{10} s \\
+ & 3 \delta_{11}^{2} s-30 \delta_{11} \delta_{10} s+45 \delta_{10}^{2} s-6 \delta_{12} \omega+30 \delta_{11} \omega-90 \delta_{10} \omega \\
& \\
c_{24}= & 396 \delta_{12} \alpha^{4} k^{4} \mu-36 \delta_{12} \alpha^{2} e k^{2}+12 \delta_{12} \delta_{11} \delta_{10} f-24 \delta_{12} \delta_{10}^{2} f \\
& +2 \delta_{11}^{3} f-24 \delta_{11}^{2} \delta_{10} f+60 \delta_{11} \delta_{10}^{2} f-40 \delta_{10}^{3} f+6 \delta_{12} \delta_{11} s \\
& -24 \delta_{12} \delta_{10} s-12 \delta_{11}^{2} s+60 \delta_{11} \delta_{10} s-60 \delta_{10}^{2} s+24 \delta_{12} \omega \\
& -60 \delta_{11} \omega+120 \delta_{10} \omega,
\end{aligned}
$$$$
c_{25}=2 \delta_{12}^{3} f-6 \delta_{12}^{2} \delta_{11} f+6 \delta_{12}^{2} \delta_{10} f+6 \delta_{12} \delta_{11}^{2} f-12 \delta_{12} \delta_{11} \delta_{10} f
$$$$
+6 \delta_{12} \delta_{10}^{2} f-2 \delta_{11}^{3} f+6 \delta_{11}^{2} \delta_{10} f-6 \delta_{11} \delta_{10}^{2} f+2 \delta_{10}^{3} f
$$$$
+3 \delta_{12}^{2} s-6 \delta_{12} \delta_{11} s+6 \delta_{12} \delta_{10} s+3 \delta_{11}^{2} s-6 \delta_{11} \delta_{10} s
$$$$
+3 \delta_{10}^{2} s-6 \delta_{12} \omega+6 \delta_{11} \omega-6 \delta_{10} \omega \text {, }
$$

$$
\begin{aligned}
c_{26}= & 216 \delta_{12} \alpha^{4} k^{4} \mu-60 \alpha^{4} \delta_{11} k^{4} \mu+12 \alpha^{2} \delta_{11} e k^{2}+6 \delta_{12}^{2} \delta_{10} f \\
& +6 \delta_{12} \delta_{11}^{2} f-36 \delta_{12} \delta_{11} \delta_{10} f+36 \delta_{12} \delta_{10}^{2} f-6 \delta_{11}^{3} f \\
& +36 \delta_{11}^{2} \delta_{10} f-60 \delta_{11} \delta_{10}^{2} f+30 \delta_{10}^{3} f+3 \delta_{12}^{2} s-18 \delta_{12} \delta_{11} s \\
& +36 \delta_{12} \delta_{10} s+18 \delta_{11}^{2} s-60 \delta_{11} \delta_{10} s+45 \delta_{10}^{2} s-36 \delta_{12} \omega \\
& +60 \delta_{11} \omega-90 \delta_{10} \omega
\end{aligned}
$$$$
\begin{aligned}
c_{27}= & 12 \delta_{12} \alpha^{4} k^{4} \mu-6 \alpha^{4} \delta_{11} k^{4} \mu+12 \delta_{12} \alpha^{2} e k^{2}-6 \alpha^{2} \delta_{11} e k^{2} \\
& +6 \delta_{12}^{2} \delta_{11} f-12 \delta_{12}^{2} \delta_{10} f-12 \delta_{12} \delta_{11}^{2} f+36 \delta_{12} \delta_{11} \delta_{10} f \\
& -24 \delta_{12} \delta_{10}^{2} f+6 \delta_{11}^{3} f-24 \delta_{11}^{2} \delta_{10} f+30 \delta_{11} \delta_{10}^{2} f-12 \delta_{10}^{3} f \\
& -6 \delta_{12}^{2} s+18 \delta_{12} \delta_{11} s-24 \delta_{12} \delta_{10} s-12 \delta_{11}^{2} s+30 \delta_{11} \delta_{10} s \\
& -18 \delta_{10}^{2} s+24 \delta_{12} \omega-30 \delta_{11} \omega+36 \delta_{10} \omega .
\end{aligned}
$$

By assuming that the coefficients of the same powers concerning $\eta$ in Eq. (25) are zero, then obtain the numbers of equations:

$$
c_{2 i}=0,(i=1,2, \cdots, 7) .
$$

Solve the numbers of these equations, then attain

$$
\delta_{12}=-\frac{6 \sqrt{10} \sqrt{\mu} k^{2} \alpha^{2}}{\sqrt{f}}, \delta_{11}=-\frac{6 \sqrt{10} \sqrt{\mu} k^{2} \alpha^{2}}{\sqrt{f}}, \delta_{10}=0 \text {, }
$$

where $\eta=e^{\alpha z}(\alpha \in \mathbb{C})$.

$$
\begin{gathered}
W_{s}\left(e^{\alpha z}\right)=-\frac{6 \sqrt{10} \sqrt{\mu} k^{2} \alpha^{2} / \sqrt{f}}{\left(e^{\alpha z}-1\right)^{2}}-\frac{6 \sqrt{10} \sqrt{\mu} k^{2} \alpha^{2} / \sqrt{f}}{e^{\alpha z}-1}, \\
W_{s}\left(e^{\alpha z}\right)=-\frac{\left(6 \sqrt{10} \sqrt{\mu} k^{2} \alpha^{2} / \sqrt{f}\right) e^{\alpha z}}{\left(e^{\alpha z}-1\right)^{2}},
\end{gathered}
$$

so, we obtain the simply periodic solutions of Eq. (14) with pole at $z=0$

$$
W_{s 10}(z)=-\frac{3 \sqrt{10} \sqrt{\mu} k^{2} \alpha^{2}}{2 \sqrt{f}}\left(\operatorname{coth}^{2} \frac{\alpha}{2} z-1\right)
$$

where $\omega=\alpha^{4} k^{4} \mu+\alpha^{2} e k^{2}$ and $s=(1 / 5)\left(\left(5 \alpha^{2} k^{2} \mu+e\right) \sqrt{10} \sqrt{f}\right.$ $/ \sqrt{\mu})$. Furthermore,

$$
\delta_{12}=-\frac{12 \alpha^{2} e k^{2}}{s}, \delta_{11}=-\frac{12 \alpha^{2} e k^{2}}{s}, \delta_{10}=-\frac{2 \alpha^{2} e k^{2}}{s},
$$

where $\eta=e^{\alpha z}(\alpha \in \mathbb{C})$.

$$
\begin{gathered}
W_{s}\left(e^{\alpha z}\right)=-\frac{12 \alpha^{2} e k^{2} / s}{\left(e^{\alpha z}-1\right)^{2}}-\frac{12 \alpha^{2} e k^{2} / s}{e^{\alpha z}-1}-\frac{2 \alpha^{2} e k^{2}}{s}, \\
W_{s}\left(e^{\alpha z}\right)=-\frac{\left(12 \alpha^{2} e k^{2} / s\right) e^{\alpha z}}{\left(e^{\alpha z}-1\right)^{2}}-\frac{2 \alpha^{2} e k^{2}}{s},
\end{gathered}
$$

so, we attain again the simply periodic solutions of Eq. (14) with pole at $z=0$

$$
W_{s 20}(z)=-\frac{3 \alpha^{2} e k^{2}}{s}\left(\operatorname{coth}^{2} \frac{\alpha}{2} z\right)-\frac{5 \alpha^{2} e k^{2}}{s},
$$

where $\omega=-\alpha^{2} e k^{2}$ and $f=0$.

By the weak $\langle 1,2\rangle$ condition, so, we introduce here the elliptic solutions by (9) with $z=0$ pole.

$$
W_{d 0}(z)=\delta_{12} \wp(z)+\delta_{10},
$$

substitute $W_{d 0}(z)$ into Eq. (14); then, we have

$$
\sum_{i=0}^{3} c_{3 i} \wp^{i}(z)=0
$$




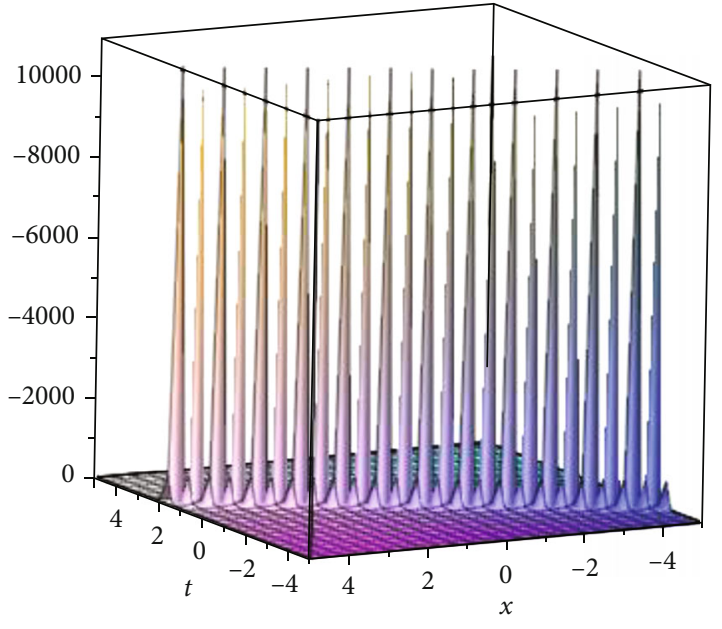

Figure 1: Perspective view of 3D graph of $W_{r, 1}(z)$ for the fixed values $\omega=2, z_{0}=0.5, \mu=1, k=1$, and $f=1$ represents the exact solutions.

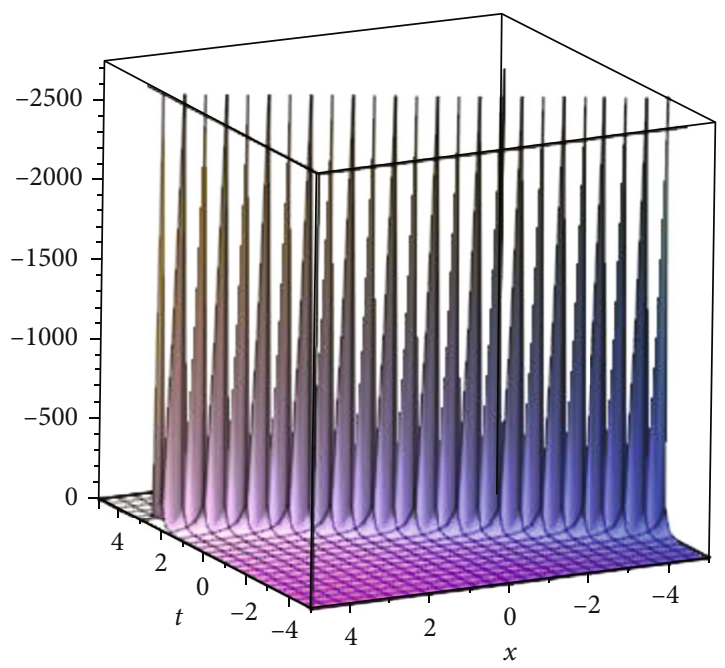

Figure 2: Perspective view of 3D graph of $W_{r, 1}(z)$ for the fixed values $\omega=2, z_{0}=-0.5, \mu=1, k=1$, and $f=1$ represents the exact solution.

where

$$
\begin{gathered}
c_{30}=-\delta_{10} \omega+\frac{1}{2} \delta_{10}^{2} s+\frac{1}{3} \delta_{10}^{3} f-\frac{1}{2} e k^{2} a g_{3}-12 \delta_{12} g_{3} k^{4} \mu, \\
c_{31}=-18 \delta_{12} g_{2} k^{4} \mu+\delta_{12} \delta_{10}^{2} f+\delta_{12} \delta_{10} s-\delta_{10} \omega, \\
c_{32}=\frac{1}{2} \delta_{12}^{2} s+\delta_{12}^{2} \delta_{10} f+6 e k^{2} \delta_{12}, \\
c_{33}=\frac{1}{3} \delta_{12}^{3} f+120 \delta_{12} \mu k^{4} .
\end{gathered}
$$

By assuming that the coefficients of the same powers concerning $\wp(z)$ in Eq. (35) are zero, then obtain the num-

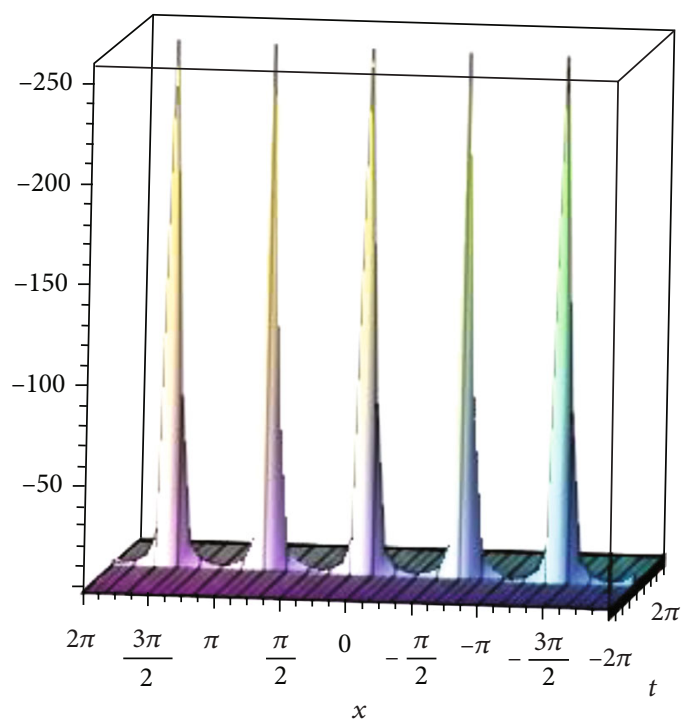

FIgURE 3: Perspective view of 3D graph of $W_{s, 1}(z)$ for the fixed values $\omega=9, z_{0}=1 / 6, \mu=1, k=1, f=1$, and $\alpha=1$ represents the exact solutions.

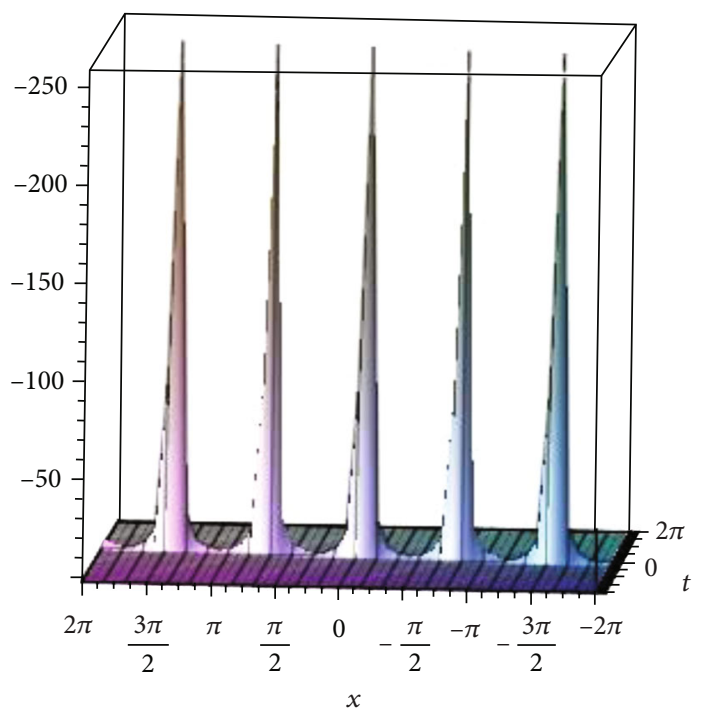

Figure 4: Perspective view of $3 \mathrm{D}$ graph of $W_{s, 1}(z)$ for the fixed values $\omega=9, z_{0}=-1 / 6, \mu=1, k=1, f=1$, and $\alpha=1$ represents the exact solutions.

bers of equations:

$$
c_{3 i}=0,(i=0,1, \cdots, 3) .
$$

Solve these equation; then, we have

$$
\delta_{12}=-\frac{6 \sqrt{10} \sqrt{\mu} k^{2}}{\sqrt{f}}, \delta_{10}=0
$$




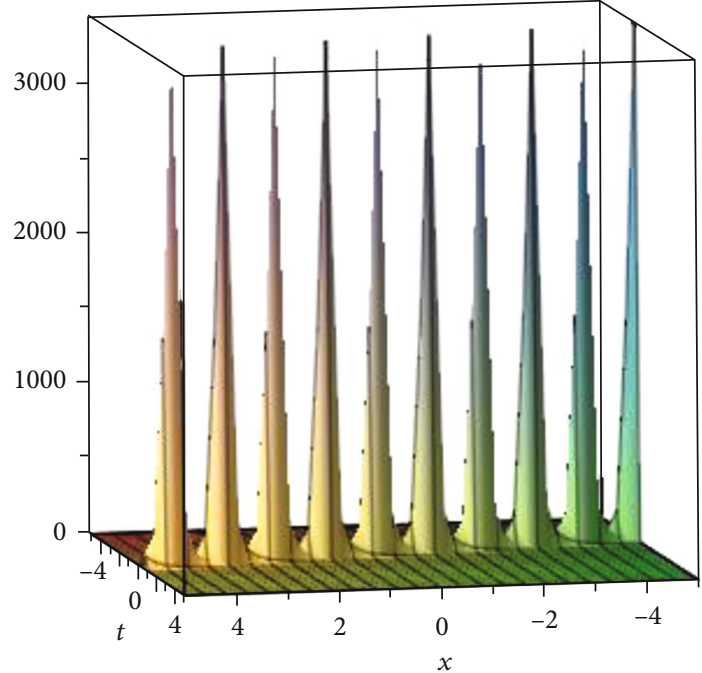

Figure 5: Perspective view of 3D graph of $W_{r, 2}(z)$ for the fixed values $\omega=5, z_{0}=1 / 3, \mu=1, k=1, s=1$, and $e=1$ represents exact solutions.

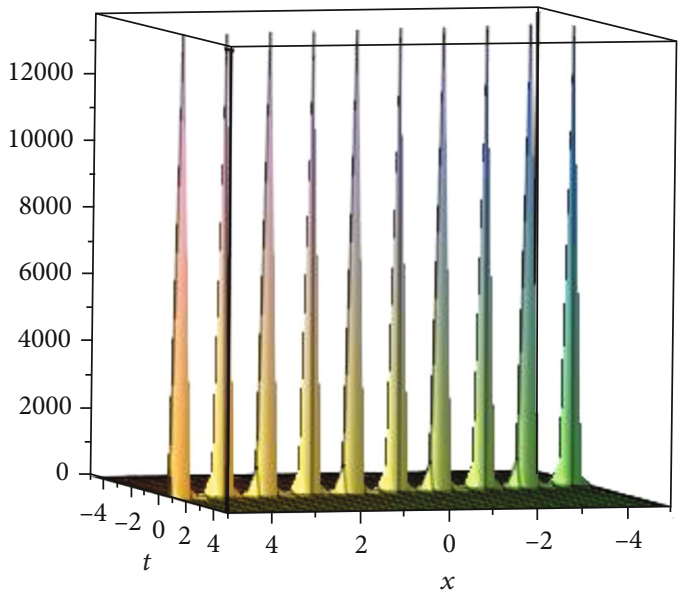

Figure 6: Perspective view of 3D graph of $W_{r, 2}(z)$ for the fixed values $\omega=5, z_{0}=-1 / 3, \mu=1, k=1, s=1$, and $e=1$ represents exact solutions.

and then we have

$$
W_{d 0}(z)=-\frac{6 \sqrt{10} \sqrt{\mu} k^{2}}{\sqrt{f}} \wp(z)
$$

hence, the elliptic general solutions of Eq. (14) with arbitrary pole are expressed such as

$$
W_{d, 1}(z)=-\frac{6 \sqrt{10} \sqrt{\mu} k^{2}}{\sqrt{f}} \wp\left(z-z_{0}\right)
$$

where $z_{0} \in \mathbb{C}$.

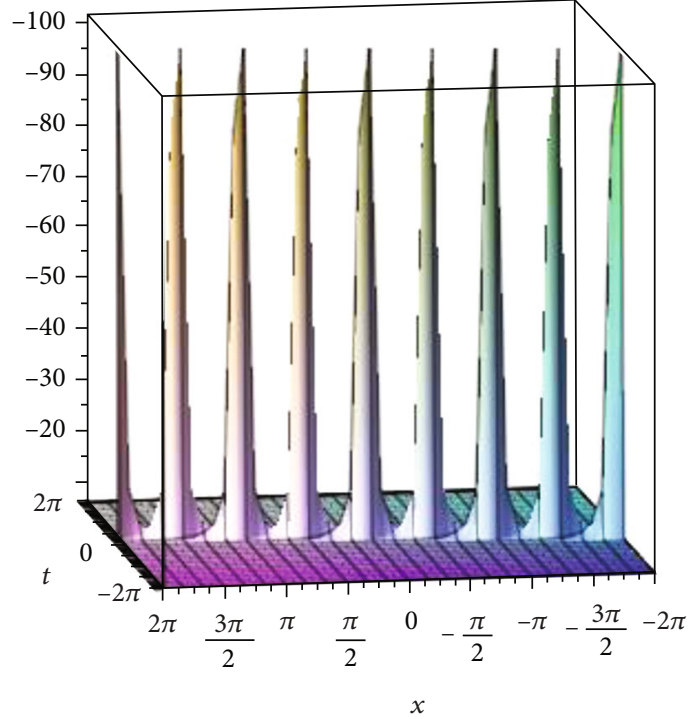

Figure 7: Perspective view of 3D graph of $W_{s, 2}(z)$ for the fixed values $\omega=6, z_{0}=1 / 8, \mu=1, k=1, s=1, e=1$, and $\alpha=1$ represents exact solutions.

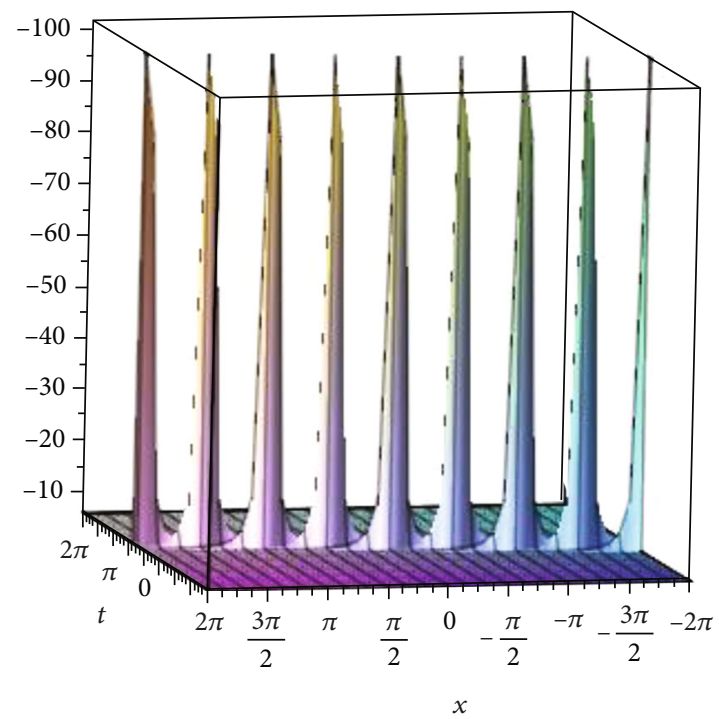

Figure 8: Perspective view of 3D graph of $W_{s, 2}(z)$ for the fixed values $\omega=6, z_{0}=-1 / 8, \quad \mu=1, \quad k=1, \quad s=1, \quad e=1$, and $\alpha=1$ represents exact solutions.

Applying the additional formula to the $W_{d, 1}(z)$, and we attain

$W_{d, 1}(z)=-\frac{6 \sqrt{10} \sqrt{\mu} k^{2}}{\sqrt{f}}\left(-\wp(z)+\frac{1}{4}\left(\frac{\wp^{\prime}(z)+G_{1}}{\wp(z)-H_{1}}\right)^{2}\right)+\frac{6 \sqrt{10} \sqrt{\mu} k^{2}}{\sqrt{f}} H_{1}$,

where $e=-24 k^{2} \mu, s=-(24 / 5) k^{2} \sqrt{\mu} \sqrt{10} \sqrt{f}, \omega=-18 g_{2} k^{4} \mu$, $G_{1}=4 H_{1}^{3}-g_{2} H_{1}-g_{3}$, and $g_{2}$ and $g_{3}$ are the arbitrary constants. 
By the above approach, so, we obtain the meromorphic solutions of Eq. (14) with arbitrary pole as mention as follows:

$$
W_{r, 1}(z)=-\frac{6 \sqrt{10} \sqrt{\mu} k^{2} / \sqrt{f}}{\left(z-z_{0}-1\right)^{2}}
$$

where $\omega=0, s=-(1 / 5)(\sqrt{f} e \sqrt{10} / \sqrt{\mu})$, and $z_{0} \in \mathbb{C}$.

$$
W_{r, 2}(z)=\frac{24 k^{2} e / s}{\left(z-z_{0}-1\right)^{2}}+\frac{6}{5} \frac{e^{2}}{s \mu}
$$

where $f=-(5 / 8)\left(\mu s^{2} / e^{2}\right), \omega=(3 / 10)\left(e^{2} / \mu\right)$, and $z_{0} \in \mathbb{C}$.

$$
W_{s, 1}(z)=-\frac{3 \sqrt{10} \sqrt{\mu} k^{2} \alpha^{2}}{2 \sqrt{f}}\left(\operatorname{coth}^{2} \frac{\alpha}{2}\left(z-z_{0}\right)-1\right)
$$

where $\omega=\alpha^{4} k^{4} \mu+\alpha^{2} e k^{2}, s=(1 / 5)\left(\left(5 \alpha^{2} k^{2} \mu+e\right) \sqrt{10} \sqrt{f} / \sqrt{\mu}\right.$ ), and $z_{0} \in \mathbb{C}$.

$$
W_{s, 2}(z)=-\frac{3 \alpha^{2} e k^{2}}{s}\left(\operatorname{coth}^{2} \frac{\alpha}{2}\left(z-z_{0}\right)\right)-\frac{5 \alpha^{2} e k^{2}}{s},
$$

where $\omega=-\alpha^{2} e k^{2}, f=0$, and $z_{0} \in \mathbb{C}$.

\section{Description about Figures}

Here, we display the exact solutions for $W_{r, 1}(z), W_{s, 1}(z)$, $W_{r, 2}(z)$, and $W_{s, 2}(z)$ by graphical phenomena as in Figures 1-8. These graphs are represented by the source of maple to persuade important results. Figures 1-8 display different multisolitary wave solutions that are obtained by different values of $z_{0}$ and $\omega$, whereas other parameters are constant.

Figures 1 and 2 indicate the exact solutions for $W_{r, 1}(z)$, adjust the values $\omega=2, z_{0}=0.5, \mu=1, k=1$, and $f=1$ and $\omega=2, z_{0}=-0.5, \mu=1, k=1$, and $f=1$.

Figures 3 and 4 indicate the exact solutions for $W_{s, 1}(z)$, adjust the values $\omega=9, z_{0}=1 / 6, \mu=1, k=1, f=1$, and $\alpha=$ 1 and $\omega=9, z_{0}=-1 / 6, \mu=1, k=1, f=1$, and $\alpha=1$.

Figures 5 and 6 indicate the exact solutions for $W_{r, 2}(z)$, adjust the values $\omega=5, z_{0}=1 / 3, \mu=1, k=1, s=1$, and $e=1$ and $\omega=5, z_{0}=-1 / 3, \mu=1, k=1, s=1$, and $e=1$.

Figures 7 and 8 indicate the exact solutions for $W_{s, 2}(z)$, adjust the values $\omega=6, z_{0}=1 / 8, \mu=1, k=1, s=1, e=1$, and $\alpha=1$ and $\omega=6, z_{0}=-1 / 8, \mu=1, k=1, s=1, e=1$, and $\alpha=1$.

\section{Comparison and Conclusion}

Khan et al. [25] represented the modified simple equation technique for the analytical treatment of generalized fifthorder KdV equation. This proposed technique provides fresh exact solutions in the area of engineering and mathematical physics. The results demonstrated the remarkable exact solutions for this technique. For this purpose, we create the com- parison between the modified simple equation technique and the extended complex approach.

We employed the extended complex technique to explore the exact analytical solutions of the generalized fifth-order $\mathrm{KdV}$ equation. The graphical phenomena are showed by setting the values of arbitrary parameters, and the graphical representations are revealed the mechanism of wave behavior, for example, Figures 1-8 depict that different multisolitary wave solutions are attained by different values of $z_{0}$ and $\omega$, whereas other parameters are constant. The extended complex approach is calculated by the source of maple software. This approach is a powerful analytical technique since it provides different new exact solutions which are indicated by the forms of rational, periodic, and elliptic function solutions. These results have been obtained by the extended complex technique to show a deeper understanding of physical structures and provide remarkable exact solutions of higher degree NPDEs.

\section{Data Availability}

The data used to support the finding of this study are mentioned in the article.

\section{Conflicts of Interest}

The authors mentioned here that they have no conflict of interests.

\section{Acknowledgments}

This work is supported by the NSFC (11901111) and Visiting Scholar Program of Chern Institute of Mathematics.

\section{References}

[1] C. Chen, X. Zhang, G. Zhang, and Y. Zhang, "A two-grid finite element method for nonlinear parabolic integro-differential equations," International Journal of Computer Mathematics, vol. 96, no. 10, pp. 2010-2023, 2019.

[2] C. Chen and X. Zhao, "A posteriori error estimate for finite volume element method of the parabolic equations," Numerical Methods for Partial Differential Equations, vol. 33, no. 1, pp. 259-275, 2017.

[3] C. Chen, K. Li, Y. Chen, and Y. Huang, "Two-grid finite element methods combined with Crank-Nicolson scheme for nonlinear Sobolev equations," Advances in Computational Mathematics, vol. 45, no. 2, pp. 611-630, 2019.

[4] C. Chen, H. Liu, X. Zheng, and H. Wang, "A two-grid MMOC finite element method for nonlinear variable-order time- fractional mobile/immobile advection-diffusion equations," Computers and Mathematics with Applications, vol. 79, no. 9, pp. 2771-2783, 2020.

[5] X. Zhang, L. Liu, Y. Wu, and Y. Cui, "A sufficient and necessary condition of existence of blow-up radial solutions for a $k$ -Hessian equation with a nonlinear operator," Nonlinear Analysis: Modelling and Control, vol. 25, pp. 126-143, 2020.

[6] X. Zhang, J. Jiang, Y. Wu, and Y. Cui, "The existence and nonexistence of entire large solutions for a quasilinear Schrödinger elliptic system by dual approach," Applied Mathematics Letters, vol. 100, p. 106018, 2020. 
[7] J. He, X. Zhang, L. Liu, Y. Wu, and Y. Cui, "A singular fractional Kelvin-Voigt model involving a nonlinear operator and their convergence properties," Boundary Value Problems, vol. 2019, no. 1, 2019.

[8] X. Zhang, J. Jiang, Y. Wu, and Y. Cui, "Existence and asymptotic properties of solutions for a nonlinear Schrödinger elliptic equation from geophysical fluid flows," Applied Mathematics Letters, vol. 90, pp. 229-237, 2019.

[9] X. Zhang, L. Liu, Y. Wu, and Y. Cui, “The existence and nonexistence of entire large solutions for a quasilinear Schrödinger elliptic system by dual approach," Journal of Mathematical Analysis and Applications, vol. 464, no. 2, pp. 1089-1106, 2018.

[10] X. Zhang, L. Liu, Y. Wu, and Y. Cui, "Existence of infinitely solutions for a modified nonlinear Schrodinger equation via dual approach," Electronic Journal of Differential Equations, vol. 2147, pp. 1-15, 2018.

[11] A.-M. Wazwaz, "A sine-cosine method for handlingnonlinear wave equations," Mathematical and Computer Modelling, vol. 40, no. 5-6, pp. 499-508, 2004.

[12] H. M. Baskonus, T. A. Sulaiman, H. Bulut, and T. Aktürk, "Investigations of dark, bright, combined dark-bright optical and other soliton solutions in the complex cubic nonlinear Schrödinger equation with $\sigma$-potential," Superlattices and Microstructures, vol. 115, pp. 19-29, 2018.

[13] H. Ahmad, T. A. Khan, and C. Cesarano, "Numerical solutions of coupled Burgers' equations," Axioms, vol. 8, no. 4, p. 119, 2019.

[14] J.-H. He, "Homotopy perturbation method: a new nonlinear analytical technique," Applied Mathematics and Computation, vol. 135, no. 1, pp. 73-79, 2003.

[15] M. Wang and X. Li, "Extended_F_-expansion method and periodic wave solutions for the generalized Zakharov equations," Physics Letters A, vol. 343, no. 1-3, pp. 48-54, 2005.

[16] J. H. He and X. H. Wu, "Exp-function method for nonlinear wave equations," Chaos, Solitons \& Fractals, vol. 30, no. 3, pp. 700-708, 2006.

[17] M. T. Darvishi, S. Arbabi, M. Najafi, and A. M. Wazwaz, "Traveling wave solutions of a $(2+1)$-dimensional Zakharov-like equation by the first integral method and the tanh method," Optik, vol. 127, no. 16, pp. 6312-6321, 2016.

[18] J. L. Hu, "A new method for finding exact traveling wave solutions to nonlinear partial differential equations," Physics Letter A, vol. 39, pp. 175-179, 2001.

[19] S. Tuluce Demiray, Y. Pandir, and H. Bulut, "Generalized Kudryashov method for time-fractional differential equations," Abstract and Applied Analysis, vol. 2014, Article ID 901540, 13 pages, 2014.

[20] E. M. E. Zayed and S. Al-Joudi, "Applications of an extended $\left(G^{\prime} / G\right)$-expansion method to find exact solutions of nonlinear PDEs in mathematical physics," Mathematical Problems in Engineering, vol. 2010, Article ID 768573, 19 pages, 2010.

[21] D. Lu and B. Hong, "New exact solutions for the $(2+1)$ dimensional generalized Broer-Kaup system," Applied Mathematics and Computation, vol. 199, no. 2, pp. 572-580, 2008.

[22] A. H. Khater, W. Malfliet, D. K. Callebaut, and E. S. Kamel, "The tanh method, a simple transformation and exact analytical solutions for nonlinear reaction-diffusion equations," Chaos, Solitons \& Fractals, vol. 14, no. 3, pp. 513-522, 2002.

[23] K. A. Khalid, K. R. Raslan, and S. E. Talaat, "Non-polynomial spline method for solving coupled Burger' equations," Compu- tational Methods for Differential Equations, vol. 3, pp. 218 230, 2015.

[24] C. Lakshmi and A. Ashish, "Numerical simulation of Burgers' equation using cubic B-splines," Nonliear Engineering, vol. 6, no. 1, pp. 61-77, 2017.

[25] K. R. Raslan, T. El Danaf, and K. A. Khalid, "An efficient approach to numerical study of the coupled-BBM system with B-spline collocation method," Communication in Mathematical Modelling and Applications, vol. 1, pp. 5-15, 2016.

[26] X. Deng, J. Cao, and X. Li, "Travelling wave solutions for the nonlinear dispersion Drinfeld-Sokolov $(\mathrm{D}(\mathrm{m}, \mathrm{n}))$ system," Communications in Nonlinear Science and Numerical Simulation, vol. 15, pp. 281-290, 2010.

[27] Y. Khan and F. Austin, "Application of the Laplace decomposition method to nonlinear. homogeneous and nonhomogenous advection equations," Zeitschrift für Naturforschung A, vol. 65, no. 10, pp. 849-853, 2010.

[28] A. R. Seadawy, "Nonlinear wave solutions of the threedimensional Zakharov-Kuznetsov-Burgers equation in dusty plasma," Physica A: Statistical Mechanics and its Applications, vol. 439, pp. 124-131, 2015.

[29] A. R. Seadawy, "Ion acoustic solitary wave solutions of twodimensional nonlinear Kadomtsev-Petviashvili-Burgers equation in quantum plasma," Mathematical Methods in the Applied Sciences, vol. 40, no. 5, pp. 1598-1607, 2017.

[30] X. Li and M. Wang, "A sub-ODE method for finding exact solutions of a generalized $\mathrm{KdV}-\mathrm{mKdV}$ equation with highorder nonlinear terms," Physics Letters A, vol. 361, no. 1-2, pp. 115-118, 2007.

[31] V. B. Matveev and M. A. Salle, "Darboux transformations and solitons," in Springer Series in Nonlinear Dynamics, p. 396, Springer-Verlag, 1991.

[32] E. Fan, "Extended tanh-function method and its applications to nonlinear equations," Physics Letters A, vol. 277, no. 4-5, pp. 212-218, 2000.

[33] M. A. Abdou, "The extended tanh method and its applications for solving nonlinear physical models," Applied Mathematics and Computation, vol. 190, no. 1, pp. 988-996, 2007.

[34] M. A. Khan, "Exact and solitary wave solutions to the generalized fifth-order KdV equation by using the modified simple equation method," Applied and Computational Mathematics, vol. 4, no. 3, pp. 122-129, 2015.

[35] R. Hedli and A. Kadem, "Exact traveling wave solutions to the fifth-order $\mathrm{KdV}$ equation using the exponential expansion method," International Journal of Applied Mathematics, vol. 50, pp. 1-6, 2020.

[36] S. Dinarvand, S. Khosravi, A. Doosthoseini, and M. M. Rashid, "The homotopy analysis method for solving the SawadaKotera and Lax's fifth-order KdV equations," Advances in Theoretical and Applied Mechanics, vol. 1, no. 7, pp. 327-335, 2008.

[37] A. Salas and J. G. Lugo, "Exact solutions for the general fifth order KdV equation by the extended tanh method," 2008, http://arxiv.org/abs/0809.2870.

[38] M. N. Alam and L. Xin, "Exact traveling wave solutions to higher order nonlinear equations," Journal of Ocean Engineering and Science, vol. 4, no. 3, pp. 276-288, 2019.

[39] D. D. Ganji and M. Abdollahzadeh, "Exact travelling solutions for the Lax's seventh-order KdV equation by sech method and rational exp-function method," Applied mathematics and Computation, vol. 206, no. 1, pp. 438-444, 2008. 
[40] W. Yuan, Y. Li, and J. Lin, "Meromorphic solutions of an auxiliary ordinary differential equation using complex method," Mathematical Methods in the Applied Sciences, vol. 36, no. 13, pp. 1776-1782, 2013.

[41] W. Yuan, Z. Huang, M. Fu, and J. Lai, “The general solutions of an auxiliary ordinary differential equation using complex method and its applications," Advances in Difference Equations, vol. 147, 2014.

[42] Y. Gu and Y. Kong, "Two different systematic techniques to seek analytical solutions of the higher-order modified Boussinesq equation," IEEE Access, vol. 7, pp. 96818-96826, 2019.

[43] Y. Gu, W. Yuan, N. Aminakbari, and J. Lin, "Meromorphic solutions of some algebraic differential equations related Painlevé equation IV and its applications," Mathematical Methods in the Applied Sciences, vol. 41, no. 10, pp. 3832-3840, 2018.

[44] Y. Gu, C. Wu, X. Yao, and W. Yuan, "Characterizations of all real solutions for the $\mathrm{KdV}$ equation and $\mathrm{W}_{R}$," Applied Mathematics Letters, vol. 107, article 106446, 2020.

[45] M. F. U. Rehman, Y. Gu, and W. Yuan, "Exact analytical solutions of nonlinear fractional Liouville equation by extended complex method," Advances in Mathematical Physics, vol. 2020, 8 pages, 2020.

[46] Y. Gu and F. Meng, "Searching for analytical solutions of the $(2+1)$-dimentional KP equation by two different systematic methods," Complexity, vol. 2019, Article ID 5162038, 9 pages, 2019. 